\title{
Primary adenocarcinoma of the jejunum and ileum: clinicopathological review of 25 cases
}

\author{
T F Lioe, J D Biggart
}

\begin{abstract}
Between 1954 and 1988 only a total of twenty five cases of primary adenocarcinoma of the small bowel (excluding periampillary tumours) were recorded at the Department of Histopathology, Belfast City Hospital. Of these, 14 tumours were located in the jejunum: the remainder arose in the ileum. The average age at presentation was $61 \cdot 3$ years and a slight female to male preponderance of 1:7:1 was noted. The adenocarcinoma arose from a preexisting villous adenoma in six cases. The overall prognosis was poor, with a five year survival of $15 \cdot 7 \%$. All the survivors had tumours located in the jejunum. The single most important prognostic indicator was the depth of tumour invasion or stage at the time of diagnosis. Tumour size and grade did not seem to correlate well with survival.

It is concluded that the rarity of these tumours and their inaccessibility hinder detection and treatment and that surgical resection is more effective than chemotherapy.
\end{abstract}

Primary adenocarcinomas of the small intestine are rare. Although the small bowel accounts for about three quarters of the length of gastrointestinal tract, malignant tumours of the small intestine only account for $1-5 \%$ of all gastrointestinal tract tumours. Most investigators $^{1-4}$ have agreed that adenocarcinomas are the most common malignant tumours of the small intestine with carcinoid tumours a close second, but others disagree. ${ }^{56}$ Between 1954 and 1988, we collected 84 cases of primary adenocarcinomas (59 cases in the duodenum and ampulla of Vater) while 49 cases of carcinoid tumours were recorded during the same period.

Duodenal and periampullary carcinomas differ clinically from jejunal and ileal carcinomas. Periampullary tumours may also arise from the bile duct or pancreatic ducts. We therefore did not include these tumours in this study but we did include tumours arising in the duodeno-jejunal flexure.

The rarity of these tumours combined with non-specific signs and symptoms can delay prompt diagnosis and treatment. Furthermore, their inaccessibility by endoscopic instruments and the limitations of radiographic techniques hinder their early detection.

\section{Methods}

Twenty six cases of primary adenocarcinoma of the small intestine, excluding duodenal and periampullary tumours, were retrieved from our files at the Belfast City Hospital between 1954 and 1988. The charts of 19 of the cases were available for study, although all the slides were reviewed. The duration of signs and symptoms, treatment, and clinical outcome were noted. The site of the tumour was checked with the surgical notes.

The mucosal origin of the tumour was sought. One tumour was excluded when the mucosal origin could not be established and the poorly differentiated tumour cells stained negatively for epithelial markers by the immunoperoxidase technique. The tumours were graded according to differentiation: grade $1=$ well, grade $2=$ moderately, and grade $3=$ poorly differentiated (figs 1a-c), depending on the degree of glandular formation and nuclear characteristics.

Well differentiated adenocarcinoma showed well formed tubules and nuclei of uniform size and shape. Whether or not crowding and stratification were evident in the nuclei the polarity was well maintained. Poorly differentiated tumours had irregular tubular structures or none at all, and tumour cells were arranged in sheets, cords, or clumps. Tumours with intermediate features were regarded as of moderate differentiation, and tumours which showed a varying degree of differentiation were graded according to the least differential area.

The extent of tumour invasion and spread was staged according to the modified Duke's classification for carcinoma of the colon: Dukes' $A$ is a tumour limited to the mucosa. Stage B1 defines tumour extending into muscularis propria but not penetrating through it; and stage B2 denotes tumour extending through the entire wall. Stage $\mathrm{Cl}$ is tumour limited to the wall with affected draining lymph nodes and stage C2 denotes tumour extending through all layers of the wall with affected nodes. Stage D denotes distant metastasis.

Other associated pathological features such as an extensive mucoid component of the tumour and adjacent mucosal atrophy were also noted.

\section{Results}

Sixteen of the 25 cases were female, giving a female:male ratio of $1 \cdot 7: 1$. All of the patients were Caucasian. Their ages at the time of 


\author{
Figure 1a Grade 1 well \\ differentiated \\ adenocarcinoma. Tubular \\ glands with basally \\ orientated nuclei. \\ (Haematoxylin and \\ eosin.) \\ Figure $1 b$ Grade 2 \\ moderately differentiated \\ adenocarcinoma. Irregular \\ tubular glands with loss of \\ nuclear polarity. \\ (Haematoxylin and \\ eosin.) \\ Figure 1c Grade 3 \\ poorly differentiated \\ adenocarcinoma. Tumour \\ cells arranged in sheets \\ with attempted gland \\ formation. (Haematoxylin \\ and eosin.)
}
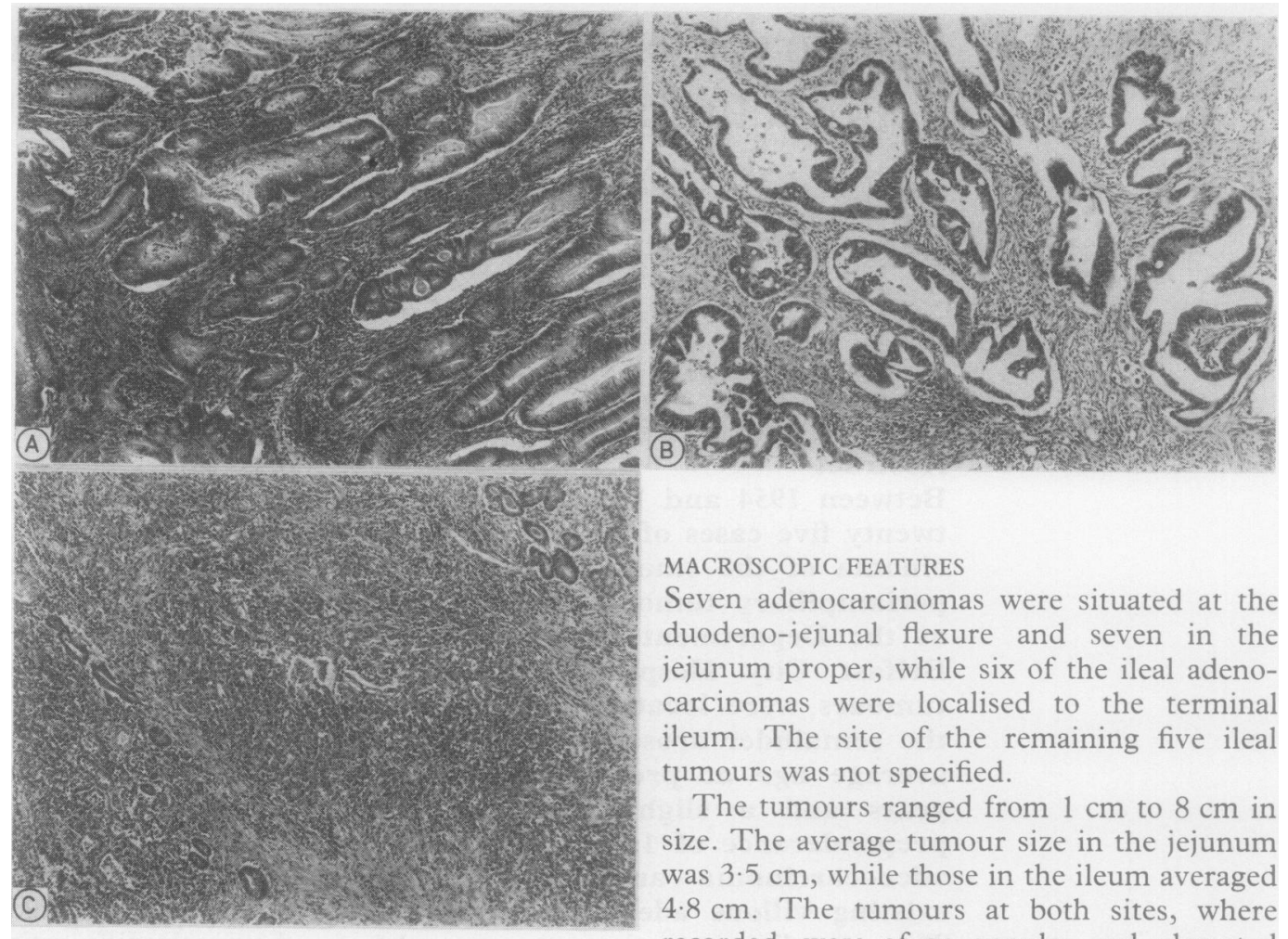

MACROSCOPIC FEATURES

Seven adenocarcinomas were situated at the duodeno-jejunal flexure and seven in the jejunum proper, while six of the ileal adenocarcinomas were localised to the termina ileum. The site of the remaining five ileal tumours was not specified.

The tumours ranged from $1 \mathrm{~cm}$ to $8 \mathrm{~cm}$ in size. The average tumour size in the jejunum was $3.5 \mathrm{~cm}$, while those in the ileum averaged $4.8 \mathrm{~cm}$. The tumours at both sites, where recorded, were of an annular and ulcerated appearance.

diagnosis ranged from 34-86 years, the average being 61.3 years and the peak age was in the eighth decade. Fourteen tumours arose from the duodeno-jejunal flexure/jejunum, while 11 were located in the ileum/terminal ileum (table 1).

\section{CLINICAL FEATURES}

The presenting complaint, or complaints, were usually related to some degree of intestinal obstruction, by far the commonest being abdominal pain. Just under $75 \%$ of our cases had epigastric pain which sometimes radiated to the back, loin, or right iliac fossa. Over two thirds also had associated nausea and vomiting. Weight loss and a change in bowel habit were seen in half the cases. None of the patients complained of melaena or had jaundice. The duration of symptoms ranged from one week to 10 months with an average of nine weeks. (One patient with adenocarcinoma of the ileum had had epigastric pain for 10 years). None of our cases had any of the familial polyposis syndromes.

In two female patients, a cholecystectomy had been performed one and three months, respectively, before an adenocarcinoma of the ileum in one and of the jejunum in the other had been dignosed.

Table 1 Age, sex, and site distribution of tumours

\begin{tabular}{lccccc}
\hline Age (years) & $\begin{array}{l}\text { Total No } \\
\text { of patients }\end{array}$ & $\begin{array}{l}\text { Duodenal-jejunal } \\
\text { flexure/jejunum }\end{array}$ & $\begin{array}{l}\text { Ileum/ } \\
\text { terminal ileum }\end{array}$ & $F$ & $M$ \\
\hline $30-39$ & 1 & 1 & & 1 & \\
$40-49$ & 3 & 2 & 1 & 2 & 1 \\
$50-59$ & 7 & 4 & 3 & 3 & 4 \\
$60-69$ & 5 & 2 & 3 & 6 & 2 \\
$70-79$ & 8 & 5 & 1 & 1 & \\
$80-89$ & 1 & 14 & 11 & 16 & 9 \\
Totals & 25 & 14 & & \\
\hline
\end{tabular}

\section{MICROSCOPIC FINDINGS}

The mucosal origin was identified in all the tumours. Most jejunal and ileal adenocarcinomas, $64 \cdot 3^{\circ}{ }_{0}$ and $63 \cdot 6^{\circ}$ o, respectively, were grade 3 . Only one jejunal tumour was well differentiated, while none of the ileal tumours was grade 1 (table 2 ).

Six of $14(42 \cdot 8 \%)$ of the jejunal adenocarcinomas showed nodal disease and distant metastasis at the time of surgical intervention, while seven of the $11(63.6 \%)$ ileal tumours were staged $\mathrm{C} 2$ and $\mathrm{D}$ (table 2 ). In six of the cases, five jejunal and one ileal, the adenocarcinoma could be seen arising from a pre-existing villous adenoma (fig 2), and in four, two cases of each, the tumour had a large mucoid component. In two of the jejunal tumours the adjacent small bowel mucosa for some distance away from the neoplastic growth, showed evidence of subtotal villous atrophy associated with crypt hyperplasia and an increase in chronic inflammatory cell infiltrate in the lamina propria, features which are suggestive of coeliac disease (fig 3 ).

\section{OTHER ASSOCIATED FINDINGS}

It has been well recognised and reported by various authors that there is a higher incidence of adenocarcinoma of the small bowel in patients with Crohn's disease, and in our series one of the tumours in the terminal ileum also had features of Crohn's disease in the adjacent bowel mucosa and wall. Concurrent neoplasms were present in three cases: one case of adenocarcinoma of the terminal ileum had a Meckel's diverticulum and carcinoid tumour; another case of terminal ileum of the colon; and a third case of ileal tumour had a colonic adenoma. tumour had a second primary adenocarcinoma 
Table 2 Comparative grades and stages of adenocarcinomas

\begin{tabular}{|c|c|c|c|c|c|c|c|c|c|c|c|}
\hline \multirow[b]{2}{*}{ Site } & \multirow[b]{2}{*}{ Total No } & \multicolumn{3}{|c|}{ Grade } & \multicolumn{6}{|c|}{ Stage } & \multirow{2}{*}{$\begin{array}{l}\text { Pre-existing } \\
\text { villous adenomo }\end{array}$} \\
\hline & & 1 & 2 & 3 & $A$ & $B 1$ & $B 2$ & $C 1$ & & $D$ & \\
\hline $\begin{array}{l}\text { Duodeno-jejunal } \\
\text { flexure/jejunum }\end{array}$ & 14 & 1 & 4 & 9 & 0 & 0 & 8 & 0 & 5 & 1 & 5 \\
\hline Terminal ileum/ileum & 11 & 0 & 4 & 7 & 0 & 1 & 3 & 0 & 4 & 3 & 1 \\
\hline
\end{tabular}

\section{TREATMENT AND OUTCOME}

All our cases were treated by surgical resection. Of the 19 cases with follow up and known clinical outcome, only three were alive for more than five years, giving a five year survival of $15 \cdot 7^{\circ}{ }_{0}$. All three survivors had adenocarcinoma of the jejunum (table 3 ). One with a grade 1 stage $B 2$ tumour survived the longest at 10 years. The third survivor with a grade 3 tumour, which had metastasised to draining lymph nodes, also remained well five years after surgery with no evidence of tumour recurrences. The remainder all died within two years of tumour recurrences. In four cases metastatic tumour deposits were noted at the time of operation in the omentum, liver, colon and ovary. None of our cases had received radiotherapy or chemotherapy as an adjuvant treatment.

\section{Discussion}

The commonest site for adenocarcinoma of the small intestine is the periampullary region. Over a period of 34 years, a total of 84 cases of primary adenocarcinoma of the small intestine were diagnosed at the Belfast City Hospital. Of these, $59\left(70^{\circ}{ }_{0}\right)$ cases were situated in the duodenum or periampullary region. Of the remaining 25 cases, 14 arose in the duodenojejunal flexure and jejunum while 11 were located in the ileum and terminal ileum. During the same period, a total of 49 cases of carcinoid tumour of the small bowel were diagnosed. These figures support the findings of other workers, that adenocarcinoma is the

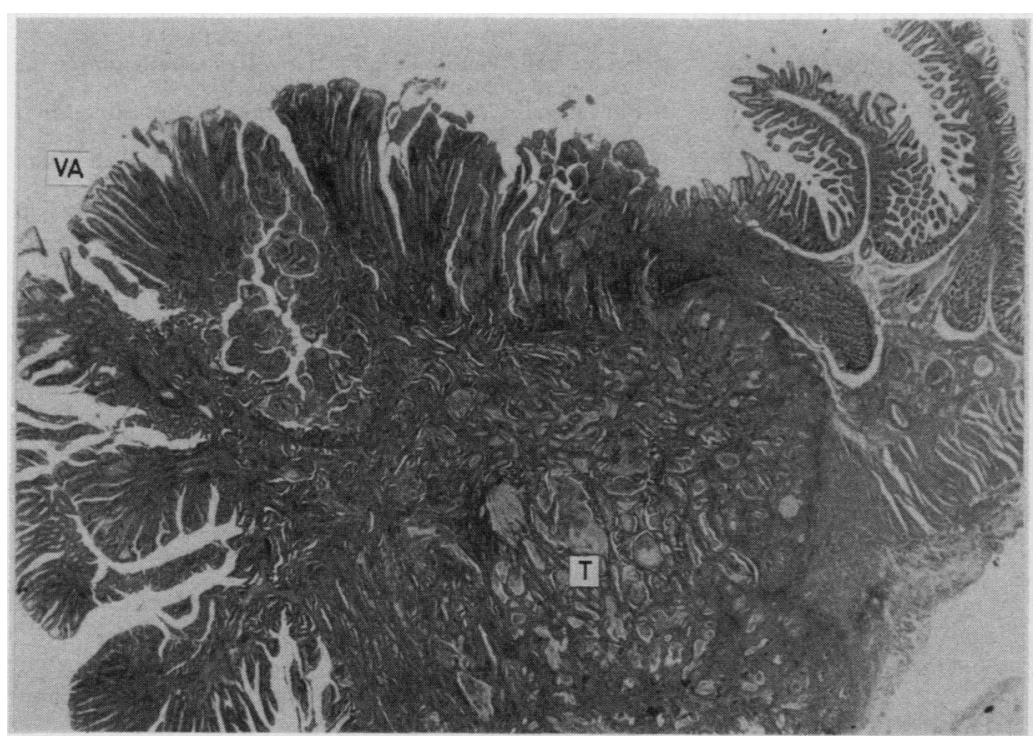

Figure 2 Adenocarcinoma ( $T$ ) arising in pre-existing villous adenoma (VA). (Haematoxylin and eosin.) commonest malignant tumour of the small bowel followed by carcinoid tumour, and that most adenocarcinomas arise in the periampullary region.

In our series of 25 cases we found a slight female preponderance, but Morgan et al noted that the reverse was true with a male to female ratio of $2: 6: 1$ in their series of 18 patients. ${ }^{2}$ Bridge et al, however, found no sex difference. ${ }^{7}$

The rarity of adenocarcinoma arising in the small bowel is largely unexplained but is most likely due to a combination of several factors. It has been postulated by Elias $e t a l^{\beta}$ and Rochlin et al, ${ }^{9}$ that adenocarcinoma of the colon occurs about 50 times more frequently than small bowel carcinomas. Factors that have been suggested as having an important role in reducing tumour formation include: (i) the dilute nature and rapid transit time of small bowel contents which reduce mucosal contact of intraluminal carcinogens compared with those of the colon ${ }^{10}$; (ii) a reduced number of bacteria, which has been associated with the carcinogenic effect of broken down bile salts as in the colon ${ }^{11}$; (iii) alkaline $\mathrm{pH}$ of the small bowel exerts a protective effect ${ }^{12}$; (iv) the presence of benzopyrene hydroxylase, which, it has been postulated, detoxifies several carcinogens ${ }^{12}$; and (v) a high concentration of secretory $\operatorname{IgA}$ antibodies which may perhaps neutralise oncogenic viruses. ${ }^{11}$

Several conditions are also known to be associated with a higher incidence of primary carcinoma in the small bowel. In our series we noted a pre-existing villous adenoma adjacent to six $(24 \%)$ adenocarcinomas, five jejunal and one ileal. Bridge et al noted a similar but less common association $(12 \%),{ }^{7}$ and Bremer et al suggested that half of all villous adenomas, undergo malignant transformation. ${ }^{13}$ Perzin et al studied 51 cases of small bowel tumours and found that 23 had tumours that contained both adenoma and carcinoma in the same lesion. ${ }^{14}$ They also found that adenoma affecting the ampulla contained carcinoma more often than lesions found elsewhere in the duodenum and small intestine.

Although $\mathrm{T}$ cell lymphomas are known to arise in the jejunal mucosa of patients with coeliac disease, there is also an uncommon association with adenocarcinoma in these patients. ${ }^{15}$ Of our 14 jejunal carcinomas, we noted in two that the adjacent small bowel mucosa distal from the main tumour mass showed features in keeping with coeliac disease. Although one of the patients also had a history of weight loss, neither was investigated for malabsorption, nor had coeliac disease been diagnosed before the discovery of the tumours.

Bridge et al reported two jejunal adenocarcinomas associated with mucosal atrophy in their series of 43 cases. ${ }^{7}$ Holmes et al described four patients with long histories of coeliac disease, three of whom were confirmed by jejunal biopsy and later progressed to develop an adenocarcinoma of the jejunum. ${ }^{9}$ In one of the adenocarcinomas of the terminal ileum in our series there was concomitant Crohn's disease. This well recognised association has been reported on many occasions. ${ }^{16-18}$ 
Table 3 Pathology details of three survivors

\begin{tabular}{llllll}
\hline $\begin{array}{l}\text { Duration } \\
\text { of symptoms }\end{array}$ & Tumour site & Tumour size $(\mathrm{cm})$ & Grade & Stage & Outcome after surgery \\
\hline 3-4 weeks & Jejunum & $3 \cdot 5$ & 1 & B2 & Alive 10 years \\
4 weeks & Jejunum & 5 & 3 & B 2 & $\begin{array}{c}\text { Alive eight years } \\
\text { Well five years, no evidence of } \\
\text { recurrence }\end{array}$ \\
6-8 months & $\begin{array}{l}\text { Duodeno-jejunal } \\
\text { flexure }\end{array}$ & $3 \cdot 5$ & 3 & C2 &
\end{tabular}

Barclay et al reported a high incidence $\left(20 \cdot 3^{\circ}{ }_{0}\right)$ of patients with adenocarcinoma of the small bowel who had a second primary cancer in other sites. ${ }^{5}$ This was not borne out in our study; in only two of our $25(8 \%)$ cases was there a second primary tumour (one carcinoid of the ileum and one carcinoma of the colon).

Adenocarcinoma of the small bowel carries an appalling prognosis. In just over half of our cases nodal disease and distant metastases were present at the time of diagnosis which usually followed a laparotomy. Only three of our 19 cases with known clinical outcome and follow up survived more than five years, giving a five year survival of $15.7 \%$. Other studies have reported a five year survival ranging from 0 $28 \%{ }^{27}$ In this series the most important prognostic factor was the depth of tumour invasion or stage at the time of diagnosis. In all three survivors in our series the tumours were located at the duodeno-jejunal flexure in one, and jejunum in the other two. We found that on average, jejunal carcinomas had a smaller size, compared with ileal tumours. This was also noted by Bridge et al. ${ }^{7}$ Perhaps patients with tumours at this site present earlier with shorter duration of symptoms. This was seen in two of the survivors who had had symptoms of four weeks duration (average in the series was nine weeks), but the other patient, however, had symptoms for six to eight months. There did not seem to be any correlation between the duration or group of symptoms and the actual site of tumour formation.

In two of the survivors the tumour was graded 3, while the other was well differentiated or grade 1. Although in one of the grade 3 survivors nodal disease was established in the resected specimen, remarkably, there was no evidence of tumour recurrence even after five

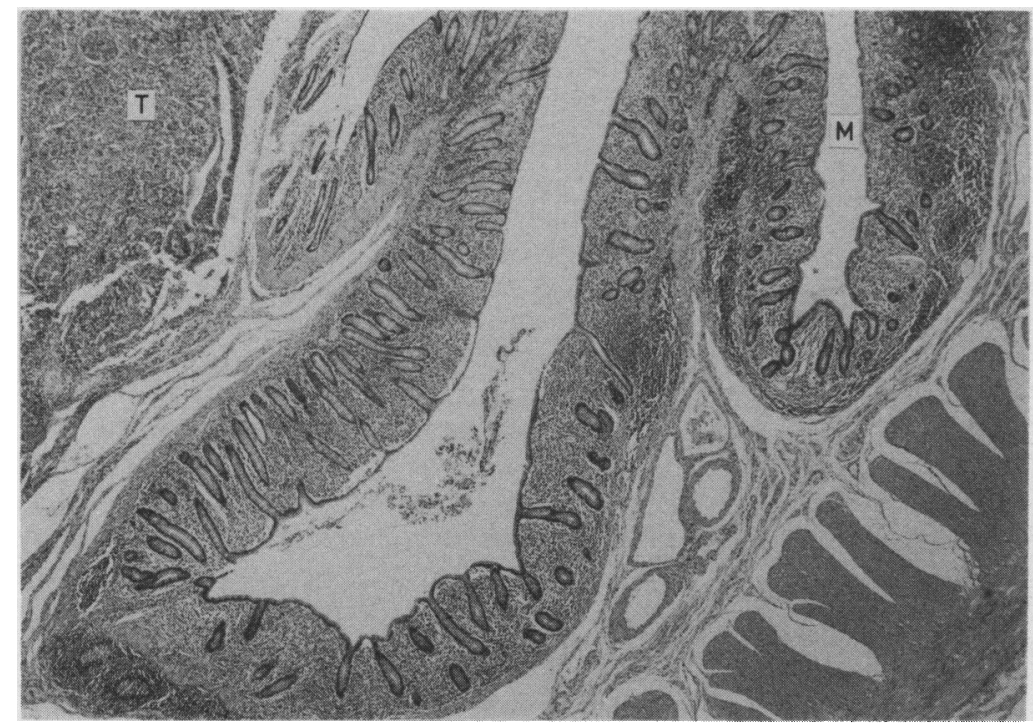

Figure 3 Mucosal atrophy $(M)$ adjacent to adenocarcinoma $(T)$. (Haematoxylin and eosin.) years. Overall, however, the tumour size and grade did not seem to correlate well with survival.

Most patients presented with features of intestinal obstruction, with epigastric pain being the most common complaint, usually associated with nausea and vomiting, weight loss, and some change in bowel habit. In two female patients a cholecystectomy was performed and the persistence of symptoms eventually led to the diagnosis of small bowel tumour. These non-specific symptoms and signs, combined with the inaccessibility of the small bowel by endoscopic instruments, often led to late diagnosis of these neoplasms. The rarity of these tumours coupled with a low index of suspicion by the clinician also hinder prompt diagnosis and lead to high mortality.

All of our patients were treated by surgical resection which seemed to be the only effective treatment. None received any adjuvant chemotherapy or radiotherapy. In the series reported by Hag et al ${ }^{19}$ and Morgan et al $^{2}$ some of their patients were given postoperative chemotherapy in the form of 5-fluorouracil, cyclophosphamide, vincristine and methyl CCNU, or a combination, but this did not seem to increase their chance of survival.

We thank Mr M Taggat for photomicrography and the secretarial staff for their assistance.

1 Reyes EL, Talley RW. Primary malignant tumours of the small intestine. Am $J$ Gastroenterol 1970;54:30-43.

2 Morgan DF, Busuttil RW. Primary adenocarcinoma of the small intestine. Am J Surg 1977;134:331-3.

3 Silberman H, Crichlow RW, Caplan HS. Neoplasms of the small bowel. Ann Surg 1974;180:157-61.

4 Mittal VK, Bodzin JH. Primary malignant tumours of the small bowel. Am J Surg 1980;140:396-9.

5 Barclay THC, Schapira DV. Malignant tumours of the smal intestine. Cancer 1983;51:878-81.

6 Awrich AE, Irish CE, Vetto RM, Fletcher WS. A 25 year experience with primary malignant tumours of the small intestine. Surg Gynecol Obstet 1980;151:9-14.

7 Bridge MF, Perzin KH. Primary adenocarcinoma of the jejunum and ileum. Cancer 1975;36:1876-87.

8 Elias WS, Lund CC. Neoplasms of the small intestine. $A m J$ Surg 1954;88:384-9.

9 Rochlin DB, Longmire WP. Primary tumours of the small intestine. Surgery 1961;50:586-92.

10 Wattenberg $\mathrm{LW}$. Carcinogen-detoxifying mechanisms in the gastrointestinal tract. Gastroenterol 1966;51:932-5.

11 Wilson JM, Melvin DB, Gray GF. Primary malignancies of the small bowel: a report of 96 cases and review of the literature. Ann Surg 1974;180:175-9.

12 Herbsman $\mathrm{H}$, Wetstein $\mathrm{L}$. Tumours of the small intestine. Curr Prob Surg 1980;17:121-82.

13 Bremer EH, Battaile WG, Bulle PH. Villous tumours of the upper gastrointestinal tract. Clinical review and report of a case. Am J Gastroenterol 1968;50:135-43.

14 Perzin $\mathrm{KH}$, Bridge MF. Adenomas of the small intestine: A clinicopathologic review of 51 cases and a study of their relationship to carcinoma. Cancer 1981;48:799-819.

15 Holmes GKT, Dunn GI, Cockel R, Brookes VS. Adenocarcinoma of the upper small bowel complicating coeliac disease. Gut 1980;21:1010-16.

16 Collier PE, Turowski P, Diamond DL. Small intestinal adenocarcinoma complicating regional enteritis. Cancer 1985;55:516-21.

17 Hawker PC, Gyde SN, Thompson H, Alan RU. Adenocarcinoma of the small intestine complicating Crohn's disease. Gut 1982;23:188-93

18 Fell J, Snooks S. Small bowel adenocarcinoma complicating Crohn's disease. JR Soc Med 1987;80:51-2.

19 Hag MM, Blumenthal BJ, Culotta RJ, Cain DN, McCleery JM. Small bowel adenocarcinoma: report of three cases and review of literature. Tex Med 1985;81:51-4. 